\title{
Nanoscale STEM/EELS and Theory Investigations of Vibronic Properties of Superlattices
}

Eric Hoglund ${ }^{1}$, Jordan Hachtel ${ }^{2}$, De-Liang Bao ${ }^{3}$, Andrew O'Hara ${ }^{3}$, Sokrates Pantelides ${ }^{3}$, Patrick Hopkins ${ }^{4}$ and James Howe ${ }^{4}$

${ }^{1}$ University of Virginia, Palmyra, Virginia, United States, ${ }^{2}$ Center for Nanophase Materials Sciences, Oak Ridge National Laboratory, United States, ${ }^{3}$ Vanderbilt University, United States, ${ }^{4}$ University of Virginia, United States

As the size of nanostructures decreases, the structural and chemical heterogeneity associated with interfaces approach the importance of the constituent materials and the local phenomena associated with interfaces can alter the macroscopic response of the material. Vibrational electron energy-loss spectroscopy (EELS), atomic-resolution imaging, and integrated differential-phase contrast (iDPC) in a scanning transmission electron microscope (STEM) provide a unique opportunity to simultaneously probe the atomic scale structure, chemical composition, and vibrational response of complex nanostructures [1], [2]. Here we combine these and other experimental techniques with density-functional-theory (DFT) calculations to describe the localized vibrational response associated with the atomic structure of interfaces in four and twenty- seven unit-cell (SL4, SL27) $\mathrm{SrTiO}_{3}-\mathrm{CaTiO}_{3}$ (STO-CTO) superlattices.

Figure 1 shows a cropped region of annular darkfield (ADF) and iDPC images along with an enlarged region-of-interest at the interface of each superlattice. Single symmetric STO oxygen columns are seen at the top of the enlarged iDPC image of SL27. In SL4 the STO oxygen columns become elliptical and some are split indicating coupled tilting to the CTO layer and an overall more uniform tilt pattern.

Off-axis vibrational EELS and DFT calculations were used to measure the spatial extent of structurally driven localization in the superlattices. We show that the layers of the superlattices systematically change with the number of unit-cells per layer in accordance with the symmetry seen in imaging, as shown in Figure 2. The emergent vibronic response of the superlattices as their layer thickness decrease below certain values demonstrates the importance for both quantitative atomic and local vibrational characterization to understand the origin of macroscopic behavior.

Acknowledgment:

Utilization of the Themis Z-STEM instrument within UVa's Nanoscale Materials Characterization Facility (NMCF) was fundamental to this work. Vibrational EELS was performed as part of a user proposal at the Oak Ridge National Laboratory (ORNL), the Center for Nanophase Materials Sciences (CNMS), which is a U.S. Department of Energy, Office of Science User Facility (J.A.H.). Work at Vanderbilt was supported by the U.S. Department of Energy, Office of Science, Basic Energy Sciences, Materials Science and Engineering Division grant No. DE-FG02-09ER46554. The authors thank Prof. Ramesh Ramamoorthy, Prof. Jayakanth Ravichandran, and Dr. Ajay Tadav for providing the thin films. The authors also thank Prof. Josh Caldwell, Dr. Thomas Beechem, Zachary Piontkowski, and Joseph Matson for their optical spectroscopy contributions to the overarching project. 
(a) 27

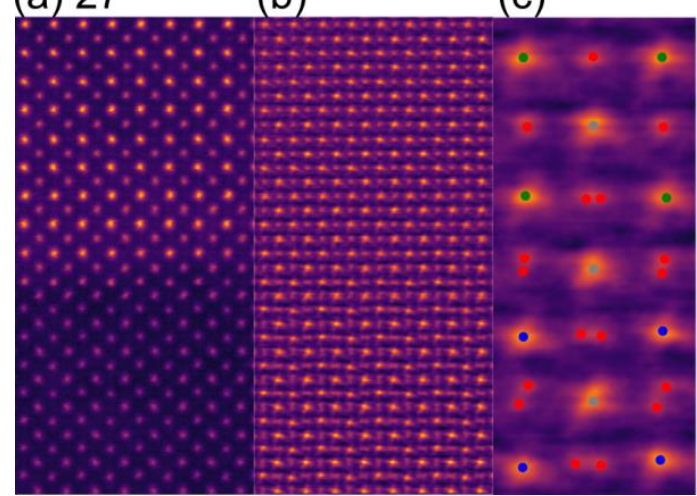

(d) 4

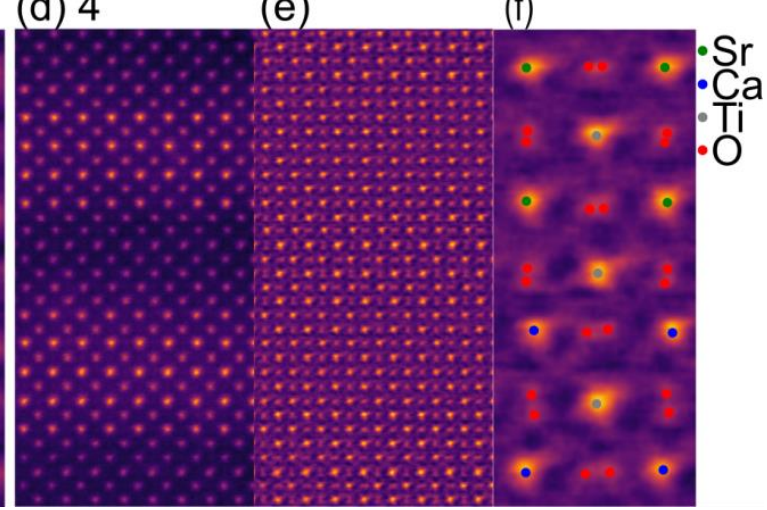

Figure 1. Figure 1. (a,d) ADF, (b,e) iDPC images, and (c,f) enlarged interface region-of-interest from iDCP images for superlattice (a-c) twenty-seven and (d-f) four.

(a)

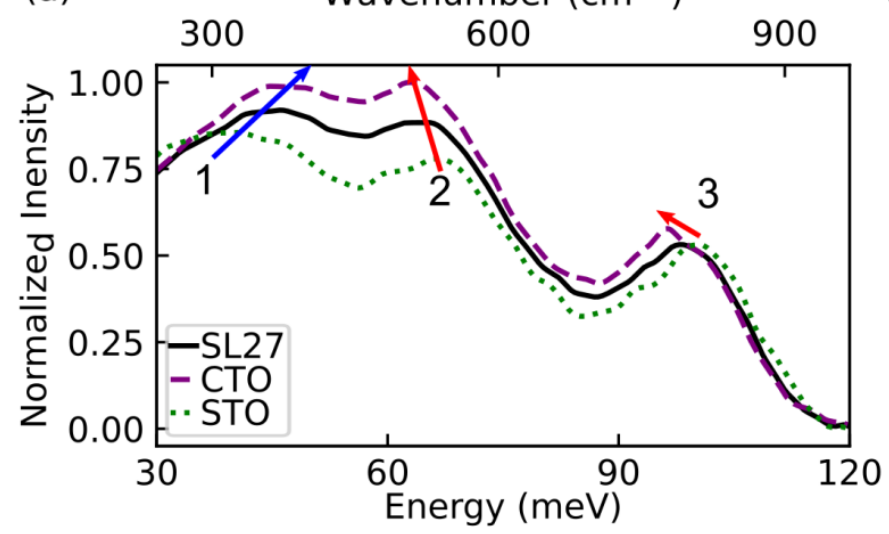

(b)

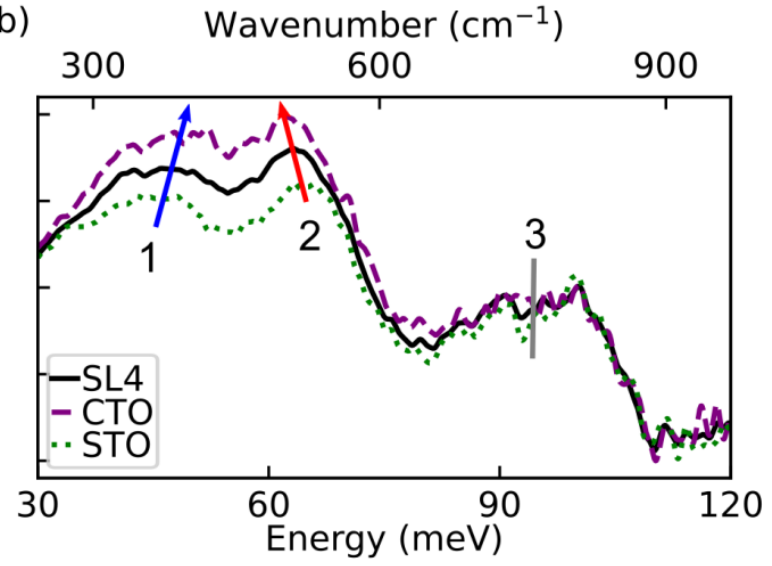

Figure 2. Figure 2. Layer-averaged off-axis vibrational EELS in (a) SL27 and (b) SL4 with arrows indicating the energy shift of peaks from STO to interface to CTO

References

[1] X. Yan, et al., Nature, $\mathbf{5 8 9} 7840$ (2021), pp. 65-69

[2] F. S. Hage, et al., Science, 3676482 (2020), pp. 1124-1127. 